\title{
Changes in cortical bone response to high-fat diet from adolescence to adulthood in mice
}

\author{
S. S. Ionova-Martin $\cdot$ J. M. Wade $\cdot$ S. Tang $\cdot$ \\ M. Shahnazari - J. W. Ager III • N. E. Lane • W. Yao • \\ T. Alliston $\cdot$ C. Vaisse $\cdot$ R. O. Ritchie
}

Received: 1 June 2010 /Accepted: 24 September 2010 / Published online: 13 October 2010

(C) The Author(s) 2010. This article is published with open access at Springerlink.com

\begin{abstract}
Summary Diabetic obesity is associated with increased fracture risk in adults and adolescents. We find in both adolescent and adult mice dramatically inferior mechanical properties and structural quality of cortical bone, in agreement with the human fracture data, although some aspects of the response to obesity appear to differ by age. Introduction The association of obesity with bone is complex and varies with age. Diabetic obese adolescents and adult humans have increased fracture risk. Prior studies have shown reduced mechanical properties as a result of high-fat diet (HFD) but do not fully address size-independent mechanical properties or structural quality, which are important to understand material behavior.
\end{abstract}

S. S. Ionova-Martin $\cdot$ J. W. Ager III $\cdot$ R. O. Ritchie

Materials Sciences Division,

Lawrence Berkeley National Laboratory,

Berkeley, CA, USA

S. S. Ionova-Martin $\cdot$ R. O. Ritchie $(\bowtie)$

Department of Materials Science and Engineering,

University of California,

Berkeley, CA, USA

e-mail: roritchie@lbl.gov

J. M. Wade $\cdot$ C. Vaisse

Diabetes Center, University of California,

San Francisco, CA, USA

S. Tang $\cdot$ T. Alliston

Department of Orthopaedic Surgery, University of California,

San Francisco, CA, USA

M. Shahnazari $\cdot$ N. E. Lane $\cdot$ W. Yao

Department of Internal Medicine, Davis Medical Center, University of California,

Sacramento, CA, USA
Methods Cortical bone from femurs and tibiae from two age groups of C57BL/6 mice fed either HFD or low-fat diet (LFD) were evaluated for structural and bone turnover changes (SEM and histomorphometry) and tested for bending strength, bending stiffness, and fracture toughness. Leptin, IGF-I, and non-enzymatic glycation measurements were also collected.

Results In both young and adult mice fed on HFD, femoral strength, stiffness, and toughness are all dramatically lower than controls. Inferior lamellar and osteocyte alignment also point to reduced structural quality in both age groups. Bone size was largely unaffected by HFD, although there was a shift from increasing bone size in obese adolescents to decreasing in adults. IGF-I levels were lower in young obese mice only. Conclusions While the response to obesity of murine cortical bone mass, bone formation, and hormonal changes appear to differ by age, the bone mechanical properties for young and adult groups are similar. In agreement with human fracture trends, adult mice may be similarly susceptible to bone fracture to the young group, although cortical bone in the two age groups responds to diabetic obesity differently.

Keywords Cortical bone - Diabetes · Fracture risk - Fracture toughness · Obesity

\section{Introduction}

The prevalence of obesity is increasing throughout the world [1]. Among many effects, obesity is a risk factor for bone fracture [2]; however, the risk of fracture is a complex one that changes over the lifetime of the individual. Obese children and adolescents tend to have an increased fracture risk $[3,4]$; non-diabetic obese adults, conversely, show the 
reverse trend [5-9]. In adults, an increased bone mineral density has been associated with obesity [5-9], and this is often cited as the primary reason for the observed reduction in fractures. In children and adolescents, however, the mechanistic picture is less clear as there are developmental consequences of obesity, such as changes in muscle development and posture control [10-12], which could markedly affect fracture risk. Additionally, activity levels may be a confounding issue, where adolescents are more likely to participate in group sports which can lead to falls and injury while adults are generally less active and may not be exposed to similar falling risks. Obesity also promotes diseases such as diabetes; indeed, fracture risk is elevated in adults with type 2 diabetes [4]. Although corresponding fracture rates for diabetic children have not been reported, reduced bone mineral content and bone size have been observed in type 1 diabetic adolescents, which implies an increased fracture risk [13].

These observations suggest an age-dependent response of bone to obesity, which are considered here by studying two groups of wild-type mice: a young group and an adult group. Fracture risk findings [3-9] would imply that adult cortical bone tends to have a more favorable response to increasing obesity than young cortical bone, although as noted, complicating factors, e.g., diabetes, activity levels, etc., may change the overall fracture risks reported by these studies.

Studies into changes in bone mineral density and content address an important aspect of bone fracture risk, but further investigation into microstructural quality and mechanical behavior, in addition to quantitative measures such as bone size and amount of mineral, may provide some insight into the changes in fracture risk throughout a lifetime. Prior work with animal models has been conducted into the question of how mechanical properties of bone are affected by both diabetic and non-diabetic obesity [14-17], but this work primarily investigated sizedependent mechanical properties (i.e., load, deflection, total energy absorbed in bend), which do not permit mechanistic delineation between the issues of the quantity vs. mechanical quality of the bone. In general, a decrease in quality of bone (i.e., reduced mechanical properties) and an increase in quantity (i.e., larger bone dimensions and bone mineral content) have been reported. To further characterize how the mechanical integrity of the tissue changes with obesity, size-independent measures such as strength, bending modulus, and toughness must also be determined [18, 19].

Many physiologic systems are affected by obesity and are important to consider in such a study. Obesity affects leptin, insulin-like growth factor I (IGF-I), and advanced glycation end-product (AGE) concentrations [7, 20, 21]. Leptin and IGF-I are both important to consider in obesity studies because they affect, and are affected by, both obesity and bone [20-22], as is non-enzymatic glycation (NEG) which can affect fracture toughness through collagen cross-linking [23-25]. Higher AGEs would also be a logical consequence of a high-fat diet, which should increase blood glucose levels, to subsequently increase the rate of NEG.

Structural changes, such as larger bone size, have been observed with obesity in both adolescents and adults [2630], and are an important characteristic to evaluate in investigating the effects of obesity on bone fracture risk. To provide further insight, macroscopic changes such as femoral length, circumference at the midshaft, and bone growth rates were performed in addition to qualitative imaging, which is a valuable tool to show bone structure changes and has been done in a prior study performed by this group [19]. By combining mechanical testing, analysis of biological factors, and structural evaluation, this study was aimed at addressing how obesity affects cortical bone at two stages in life, adolescence and adulthood, in an effort to further understand what factors influence fracture risk throughout life.

\section{Methods}

\section{Animals}

All protocols were approved by the UCSF Institutional Animal Care and Use Committee and performed according to federal guidelines for the care and use of animals in research. Thirty 3-week-old and 28 15-week-old C57BL/6 male mice were fed a high-fat diet (Research Diets HighFat Diet $60 \mathrm{kcal} \%$ fat, $20 \mathrm{kcal} \%$ carbohydrate, $20 \mathrm{kcal} \%$ protein) ( $n=15$ young and $n=14$ adult, termed "yHFD" and "aHFD" groups, respectively) or low-fat diet (Research Diets Low-Fat Diet $10 \mathrm{kcal} \%$ fat, $70 \mathrm{kcal} \%$ carbohydrate, $20 \mathrm{kcal} \%$ protein) ( $n=15$ young and $n=14$ adult, termed "yLFD" and "aLFD" groups, respectively) for a diet duration of 16 weeks. All mice, grouped in cages of five animals each, were maintained under controlled temperature and photoperiod ( $12 \mathrm{~h}$ light, $12 \mathrm{~h}$ dark) with food and water provided ad libitum. After sacrifice, all femora and tibiae were isolated, wrapped in gauze soaked with Hanks' Balanced Salt Solution (HBSS), and frozen at $-20^{\circ} \mathrm{C}$ until testing. Femora were used for mechanical testing: the left tibiae were used for histomorphometry and the right tibiae for AGE accumulation quantification.

\section{Body composition}

Body weight was measured starting on postnatal day 22 for the young mice and postnatal day 106 for the adult mice. All mice were weighed at 2-week intervals throughout the 
study and once prior to sacrifice. Fat and lean body mass (FBM and LBM), percent fat, whole-body areal bone mineral density (aBMD), and bone mineral content (BMC) were determined at the completion of the study by dual-energy X-ray absorptiometry (DXA), as instructed by the manufacturer (Lunar PIXImus mouse densitometer).

\section{Blood collection}

At the end of week 16 of the study, mice were decapitated within $30 \mathrm{~s}$ of handling. Blood was collected in tubes containing ethylene-diaminetetraacetic acid (EDTA) and plasma was immediately separated by centrifugation and frozen at $-80^{\circ} \mathrm{C}$

\section{Blood glucose test}

Blood glucose levels were measured from the tail vein using an Ascensia ELITE XL blood glucose meter. The fasting glucose measurement at age 19 and 31 weeks, respectively, was performed after overnight fasting in the last week of the study (week 16).

\section{Leptin level measurement}

Serum leptin levels were measured using a Crystal Chem Inc. Mouse Leptin ELISA kit according to the manufacturer's instructions as previously reported [19]. Both intraand inter-sample coefficients of variation for this test are $10 \%$.

\section{IGF-I level measurement}

Serum IGF-I levels were measured using an Immunodiagnostic Systems Inc. Mouse/Rat IGF-I ELISA kit according to the manufacturer's instructions as previously reported [19]. Both intra- and inter-sample coefficients of variation for this test are $7-8 \%$.

\section{Bone histomorphometry measurements}

Dynamic bone histomorphometric measures were obtained from the tibial midshaft of each animal. Mice were injected with $10 \mathrm{mg} / \mathrm{kg}$ calcein 1 and 6 days before sacrifice. At termination, tibiae were removed and fixed in $10 \%$ neutral phosphate-buffered formaldehyde for $24 \mathrm{~h}$. Tibial middiphyseal regions were cut out using a precision saw (Isomet 1000; Buehler, Lake Bluff, IL, USA) and dehydrated in increasing concentrations of ethanol and embedded undecalcified in methylmethacrylate. Transverse sections ( $40 \mu \mathrm{m}$ thick) of tibial cortex were cut at tibiafibula junction using a diamond wire saw (Well 3241, Norcross, GA, USA). The sections were cover-slipped with
Eukitt (Calibrated Instruments, Hawthorne, NY, USA) and mounted unstained for visualization under fluorescent microscopy (Eclipse E400; Nikon, Japan) for quantitative morphometry using image analysis software (Bioquant Image Analysis Corporation, Nashville, TN, USA). Endocortical and periosteal measurements included single- and double-labeled perimeter and interlabel width, which were used to calculate the mineralizing surface (MS/BS), mineral apposition rate (MAR), and bone formation rate (BFR) at both the endocortical and periosteal bone surfaces according to the standard guidelines previously published for bone histomorphometry [31]. For those samples not displaying a double label, a minimum MAR was assigned $(0.5 \mu \mathrm{m} /$ day $)$ and was used to calculate BFR.

Quantification of advanced glycation end-product accumulation

A fluorometric assay was performed in order to evaluate the extent of AGEs in HFD and LFD bone. The tibial midshafts were demineralized using EDTA and confirmed using contact radiographs. The demineralized bone samples were then hydrolyzed using $6 \mathrm{~N} \mathrm{HCl}\left(24 \mathrm{~h}, 110^{\circ} \mathrm{C}\right)$. AGE content was determined using fluorescence readings taken using a microplate reader at the excitation wavelength of $370 \mathrm{~nm}$ and emission wavelength of $440 \mathrm{~nm}$. These readings were standardized to a quinine-sulfate standard and then normalized to the amount of collagen present in each bone sample. The amount of collagen for each sample was determined based on the amount of hydroxyproline, the latter being determined using a chloramine-T colorimetric assay that recorded the absorbance of the digested samples against a hydroxyproline standard at the wavelength of $585 \mathrm{~nm}[32]$.

\section{Mechanical testing}

Size-dependent measures such as failure load and energy absorption do not account for changes in the bone crosssection area, thereby confounding the effects of bone quality and quantity. To understand the mechanical integrity of the bone and its resistance to fracture, size-independent mechanical properties (yield and maximum stresses, stiffness, and fracture toughness ${ }^{1}$ ) also need to be measured

\footnotetext{
${ }^{1}$ Strength, defined by the yield stress at the onset of permanent deformation or maximum strength at the peak load before fracture, is a measure of the force/unit area that the bone can withstand. Stiffness is related to the elastic modulus and defines the force required to produce a corresponding elastic deformation (elastic strain). The fracture toughness measures resistance to fracture of a material. However, the overall bone fracture risk of an individual will be a function of the bone quantity in addition to such measures of bone quality.
} 
$[19,33]$ as part of a larger plan of study which includes bone distribution and bone quantity measures. Prior to testing, the femora were thawed in room-temperature HBSS, and the size and geometry of all samples were measured with calipers. The left femora were tested in unnotched three-point bending to evaluate bending strength and stiffness. The right femora were tested in notched threepoint bending to assess the fracture toughness. For toughness testing, the femoral shaft was sharply notched in the mid-diaphyseal region through the posterior wall using the method described by Ritchie et al. [33]. Notches were sharpened by polishing in $1-\mu \mathrm{m}$ diamond paste with a razor blade to a root radius of $\sim 5-10 \mu \mathrm{m}$. Notched and unnotched femora were placed in a three-point bending rig such that the posterior side was in tension and the anterior was in compression. Femora were submerged in HBSS at $37^{\circ} \mathrm{C}$ for $1 \mathrm{~min}$ to acclimate, then tested in the same environment at a displacement rate of $0.001 \mathrm{~mm} / \mathrm{s}$ until fracture (EnduraTec Elf 3200, BOSE). Broken halves were then dehydrated and the fracture surfaces examined in an environmental SEM (JEOL JSM-6430 ESEM, Hitachi America). The femoral cross-sectional area and second moment of inertia were computed from fracture surface images. Notch half-crack angles were determined in the SEM from the fracture surface using techniques described in ref. [33]. Stresses and strains were computed in accordance with the methods described by Akhter et al. [34]. The yield strength $\left(\sigma_{y}\right)$ was determined as the stress at $0.2 \%$ plastic strain, and maximum strength $\left(\sigma_{u}\right)$ as the stress at peak load $\left(P_{u}\right)$. Bending stiffness $(E)$ was calculated as the slope of the linear region of the stress-strain curve. Fracture toughness $\left(K_{c}\right)$ values were defined at the onset of unstable fracture, i.e., at the point of instability, using the procedures described in ref. [33] for the toughness evaluation of small animal bone.

\section{Scanning electron microscopy}

Scanning electron microscopy (SEM) was performed to evaluate structural differences at the tissue level near the fracture surface on the medial and lateral sides of the femur. After mechanical testing, three samples each from the four study groups were mounted in Buehler Epoxycure Resin (Buehler) and the surface polished to $0.05 \mu \mathrm{m}$ with a diamond polishing suspension, coated with carbon, then imaged in an SEM (Philips XL30 ESEM-FEG; FEI Company) operating at $10 \mathrm{kV}$ in back-scattered mode as previously reported [19].

Statistical analysis

Measured values are presented as mean \pm standard deviation. Two-tailed independent sample Student's $T$ tests were executed (StatPlus:mac LE.2009) to determine differences in measured variables between the LFD and HFD groups for each age group. As the young and adult study groups were considered to be independent from each other, we did not test for changes among all groups, but rather investigated whether obesity in a particular age group had an effect on bone properties. Differences were considered to be significant at $p<0.05$. Correlation analysis was performed within each group (LFD and HFD) to identify trends that might be diet-independent. To mitigate the risk of type I errors, related measurements that were highly and positively correlated were grouped together and given a composite score (sum of Z-scores). For those measures which did not correlate to similar measurements $\left(\sigma_{u}, P_{u}\right)$ or were conceptually unique $\left(K_{c}, \mathrm{aBMD}\right)$, the Z-score for that measurement was used in the analysis without any modification. Correlation analysis was performed between scores of predictive measures (mineral density, composite bone size score, and two body composition measures) and mechanical property outcomes (composite strength and modulus score, fracture toughness, and load measures).

\section{Results}

Metabolic phenotype of experimental animals

Figure 1 summarizes the results of the weight and hormone changes in this study. Both HFD groups were significantly heavier than their LFD counterparts, with the aHFD group being $52.7 \%$ heavier than the aLFD group and the yHFD group being $44.2 \%$ heavier than the yLFD group ( $p<$ 0.0001 for both). Unsurprisingly, fat body mass (FBM) was $192 \%$ and $229 \%$ greater in adult and young HFD, respectively, compared to aLFD and yLFD $(p<0.0001)$. Lean body mass (LBM) did change slightly $(15 \%$ larger in both yHFD and aHFD compared to their respective age controls, $p<0.0001$ ); this change was likely a contributing factor to the results observed.

Blood glucose tests indicated that the obese groups were likely diabetic. Blood glucose levels in the obese groups

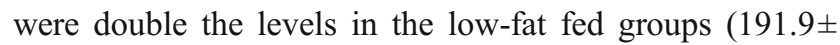
$41.1 \mathrm{mg} / \mathrm{dl}$ in aHFD vs. $99.4 \pm 29.8 \mathrm{mg} / \mathrm{dl}$ in aLFD, $p<$ $0.001 ; 187.7 \pm 39.1 \mathrm{mg} / \mathrm{dl}$ in yHFD vs. $97.7 \pm 16.3 \mathrm{mg} / \mathrm{dl}$ in yLFD, $p<0.001)$. This result is also not surprising as the $\mathrm{C} 57 \mathrm{Bl} / 6$ mouse strain is known to be susceptible to diabetes on high-fat diets.

There was a $16 \%$ increase in the serum leptin concentration in aHFD vs. aLFD, and a $235 \%$ increase in yHFD vs. yLFD $(p>0.05)$. Although not significant due to large variations, the increasing trend in serum leptin concentration is in agreement with prior studies showing that serum levels of leptin increase with obesity. 


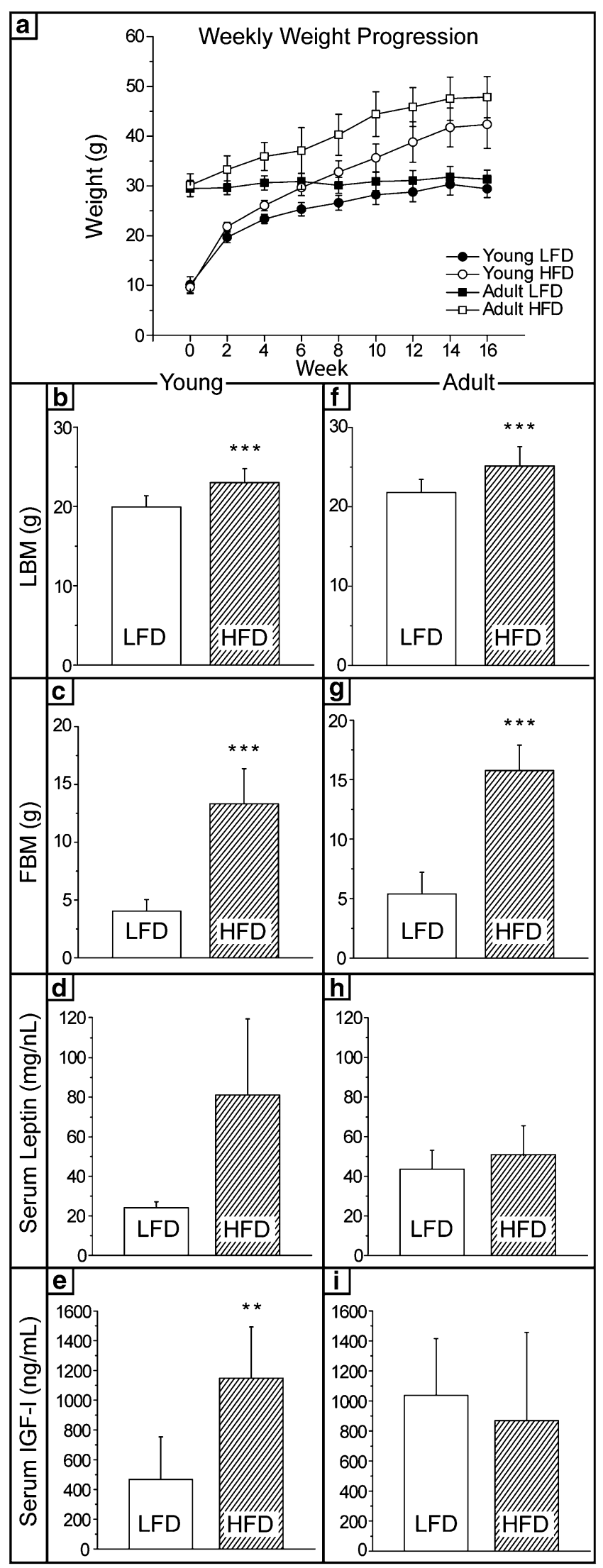

Fig. 1 Body composition, serum leptin concentration, and IGF-I concentration. a Average weekly weights of LFD and HFD groups. Horizontal axis is progression of study in weeks; $\mathbf{b}$ young and $\mathbf{f}$ adult lean body mass; $\mathbf{c}$ young and $\mathbf{g}$ adult fat body mass for LFD and HFD groups at conclusion of study; $\mathbf{d}$ young and $\mathbf{h}$ adult serum leptin concentration (mean $\pm \mathrm{SE}$ ) at conclusion of study; e young and $\mathbf{i}$ adult serum IGF-I concentrations at the conclusion of study. Both lean body mass and fat body mass increased, but signficant increase in IGF-I concentration are only observed for the yHFD group. yLFD $n=15$, yHFD $n=15$, aLFD $n=13$, aHFD $n=14\left({ }^{* *} p<0.01,{ }^{* * *} p<0.001\right)$

IGF-1 is well known to be associated with obesity as well as with greater bone size; therefore, serum IGF-1 levels were characterized in each experimental group. The insulin-like growth hormone IGF-I concentration was $145 \%$ larger in yHFD vs. yLFD $(p<0.01)$.

Bone densitometry: bone mineral content but not density smaller with high-fat diet

Figure 2 outlines the results of bone densitometry measurements performed using DXA scanning at the conclusion of the study. BMC was $12.5 \%$ lower for yHFD vs. yLFD, and a decreasing but non-significant trend was observed in the adult group as well. Whole-body areal BMD (aBMD) was unaffected in both age groups, as was femoral aBMD. The most significantly affected mineral measure was spinal aBMD, which was reduced by $18 \%$ in aHFD and $9 \%$ in yHFD compared to their LFD controls, implicating an increased spinal fracture risk at both age points. Although vertebral effects were not a part of this study, previous work by Zernicke et al. [16] found smaller L6 ash content in rats fed a high-fat-sucrose diet over 2 years.

Bone geometry: cortical bone size effect reversed with age

With respect to the measurements of bone size, femoral thickness in aHFD was smaller vs. aLFD $(p<0.01)$, likely due to reduced endocortical bone turnover as measured by dynamic histomorphometry. yHFD showed an increase in femoral diameter compared to yLFD $(p<0.01)$, as summarized in Fig. 3.

Bone histomorphometry measurements: periosteal and endosteal responses differ with diet

Total cross-sectional area did not change significantly for either age group but mean cortical width was $5 \%$ smaller in yHFD vs. yLFD $(p<0.05)$. The bone marrow cavity area was $17 \%$ larger in yHFD vs. yLFD $(p<0.05)$, which is in agreement with the cortical thickness finding and suggests larger levels of endocortical resorption in yHFD. The adult marrow area trended larger in HFD as well but this change was not significant. The endocortical bone 


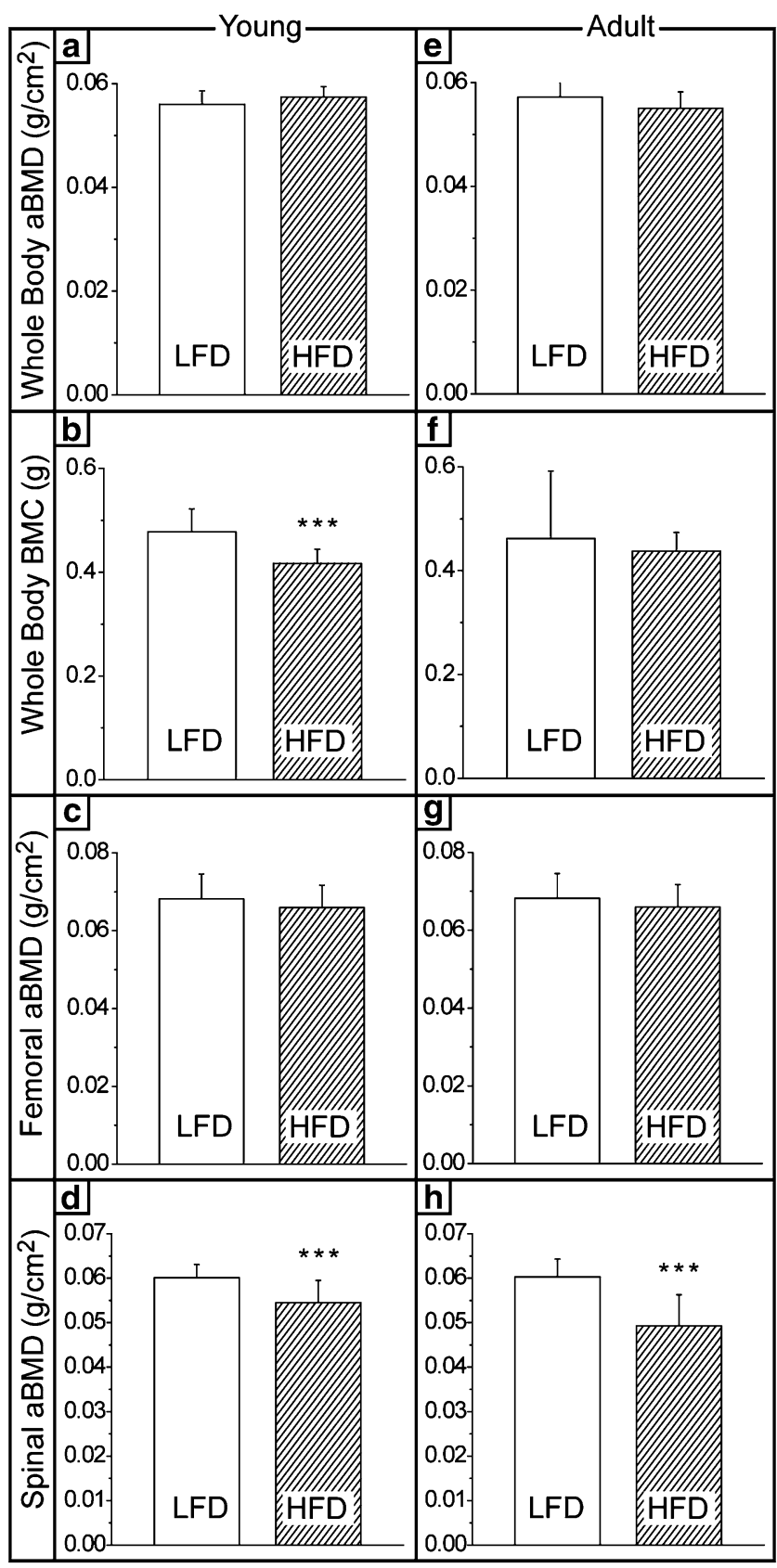

Fig. 2 Bone mineral. a Young and e adult whole-body bone mineral density (aBMD) is unchanged in HFD; $\mathbf{b}$ young and $\mathbf{f}$ adult wholebody areal bone mineral content (BMC) is lower for the yHFD vs. yLFD, which is likely due to reduced spinal aBMD. c Young and $\mathbf{g}$ adult areal bone mineral density of the femora are unchanged; $\mathbf{d}$ young and $\mathbf{h}$ adult areal bone mineral density of the spine are reduced for HFD despite increasing weight, leptin, and IGF-I. yLFD $n=15$, yHFD $n=15$, aLFD $n=13$, aHFD $n=14\left({ }^{* * *} p<0.001\right)$

formation rate (BFR) was unchanged in both age groups; however, periosteal BFR was higher in both age groups ( $p$ $<0.05$ ). Aging may have differential effects on endocortical and periosteal response to HFD, and while the former decreases the latter may increase. These results are in

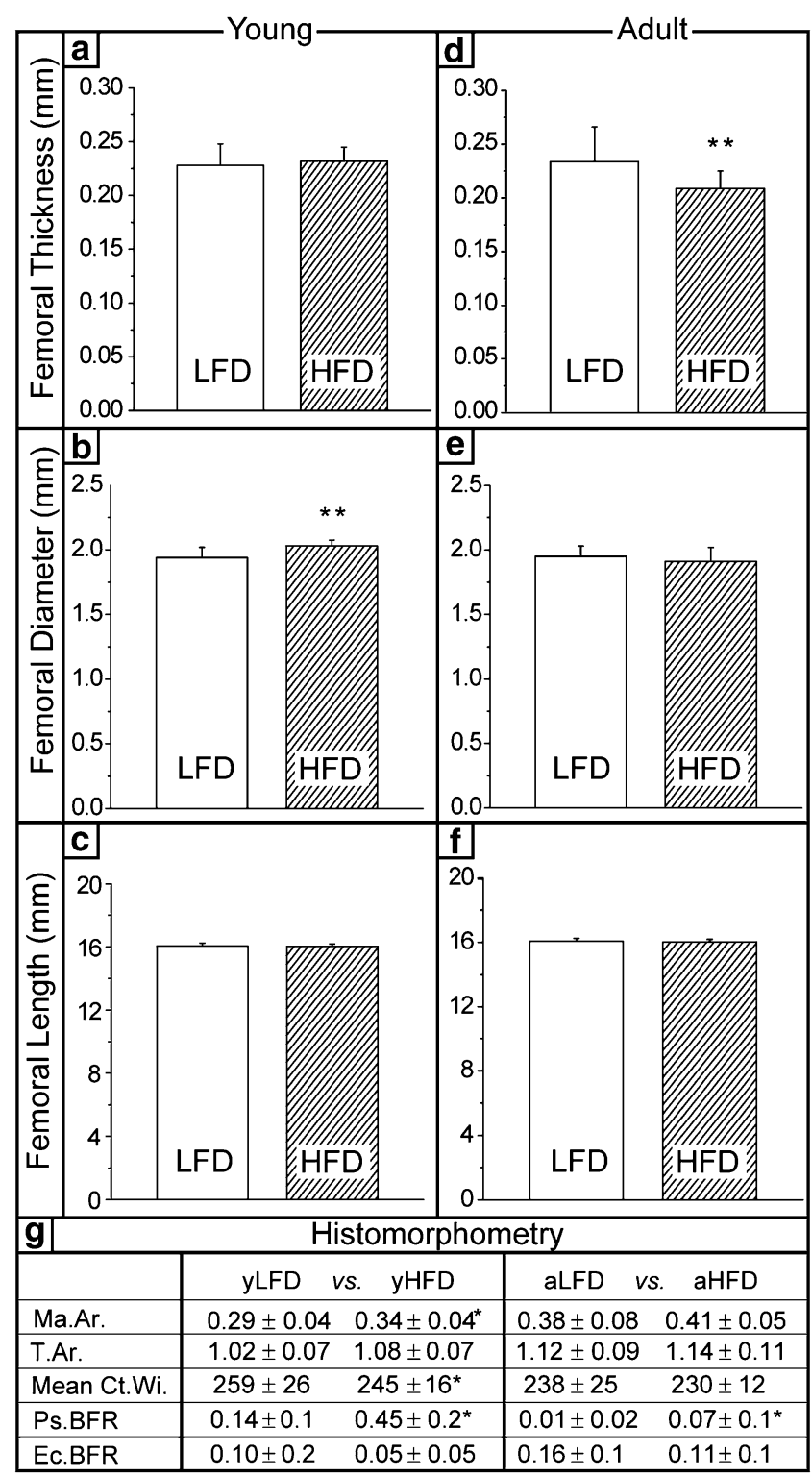

Fig. 3 Cortical bone size. a Young and $\mathbf{d}$ adult cortical thickness is reduced in adults only; $\mathbf{b}$ young and $\mathbf{e}$ adult femoral diameters are increased in yHFD vs. yLFD; $\mathbf{c}$ young and $\mathbf{f}$ adult femoral lengths are unchanged. g Histomorphometry results: $M a . A r$. marrow area $\left(\mathrm{mm}^{2}\right)$, $T . A r$. total cros-sectional area $\left(\mathrm{mm}^{2}\right)$, Mean $C t$.Wi. mean cortical width $(\mu \mathrm{m}), P s . B F R$ and Ec.BFR periosteal and endocortical bone formation rate $\left(\mu \mathrm{m}^{3} / \mu \mathrm{m}^{2} / \gamma\right)$. The general trend in the bone size data points to decreasing bone size in adults and increasing bone size in young obese mice compared to LFD, as well as a shift from periosteal activity to endosteal activity with age. yLFD $n=15$, yHFD $n=15$, aLFD $n=13$, aHFD $n=14\left({ }^{*} p<0.05,{ }^{* *} p<0.01,{ }^{* * *} p<0.001\right)$

agreement with prior aging studies even where obesity is not a factor; an effect that has been shown to occur independently of diet where increasing periosteal apposition is coupled with increasing endocortical remodeling with age [35]. 
Fig. 4 Cortical bone quality: whole-bone and tissue-level mechanical property measurements. a Young and $\mathbf{f}$ adult bending modulus; $\mathbf{b}$ young and $\mathbf{g}$ adult maximum load; $\mathbf{c}$ young and $\mathbf{h}$ adult yield stress; $\mathbf{d}$ young and $\mathbf{i}$ adult max stress; $\mathbf{e}$ young and $\mathbf{j}$ adult fracture toughness. Measured size-independent mechanical properties were significantly decreased for HFD group vs. LFD groups (modulus, yield and maximum stress, and fracture toughness); these parameters are an indication of bone tissue quality. Size-dependent measures which address whole-bone behavior (specifically, load) also declined for HFD at both ages, likely due in part to modest bone size changes, as bone size was not able to compensate for poor mechanical quality. yLFD $n=15$, yHFD $n=15$, aLFD $n=13$, aHFD $n=14 \quad\left({ }^{*} p<0.05\right.$; $\left.{ }^{* *} p<0.01\right)$

Accumulation of AGEs greater with diabetic obesity

The accumulation of AGEs were fluorimetrically quantified in the decalcified cortex of the HFD and LFD groups. AGEs are $31 \%$ higher in aHFD (42.8 $\pm 7.6 \mathrm{ng}$ quinine/mg collagen) vs. aLFD $(56.1 \pm 9.2 \mathrm{ng} / \mathrm{mg}, p<0.001)$ and $6 \%$ higher in yHFD vs. yLFD $(41.3 \pm 5.5 \mathrm{ng} / \mathrm{mg}$ vs. $39.1 \pm$ $8.7 \mathrm{ng} / \mathrm{mg}$, respectively, $p>0.05)$.

Mechanical testing: mechanical properties compromised with diabetic obesity

Overall, mechanical properties of cortical bone are compromised by diabetic obesity in both young and adult groups, as summarized in Fig. 4. Compared to the control groups, the yield strength of the bone was unchanged in aHFD ( $9 \%$ less, not significant), but was $17 \%$ less in yHFD $(p<0.01)$; corresponding maximum strengths were $15 \%$ less in aHFD $(p<0.05)$ and $26 \%$ less in yHFD $(p<0.01)$. The bending modulus was $18 \%$ less in aHFD and $32 \%$ less in yHFD $(p<0.01)$; fracture toughness, $K_{c}$, values were $21 \%$ less in aHFD $(p<0.05)$, but unchanged in yHFD ( $8 \%$ higher, not significant). Finally, the maximum loads sustained by the bone were $22 \%$ less in aHFD $(p<0.01)$ and $12.5 \%$ less in yHFD $(p<$ 0.05 ). These results indicate a profound reduction in mechanical quality and performance of the bone with diabetic obesity.

Structural characterization: poor mineral organization and lamellar alignment of cortical bone in diabetic obese mice

SEM was performed on cross-sections of femora near the fracture surface to evaluate lamellar-level structural changes. Changes in structure were most apparent at the posterior site (Fig. 5). In both the young and adult groups, the HFD bone showed marked areas of lamellar disorganization, whereas a similar area in the LFD mice appeared well-ordered.

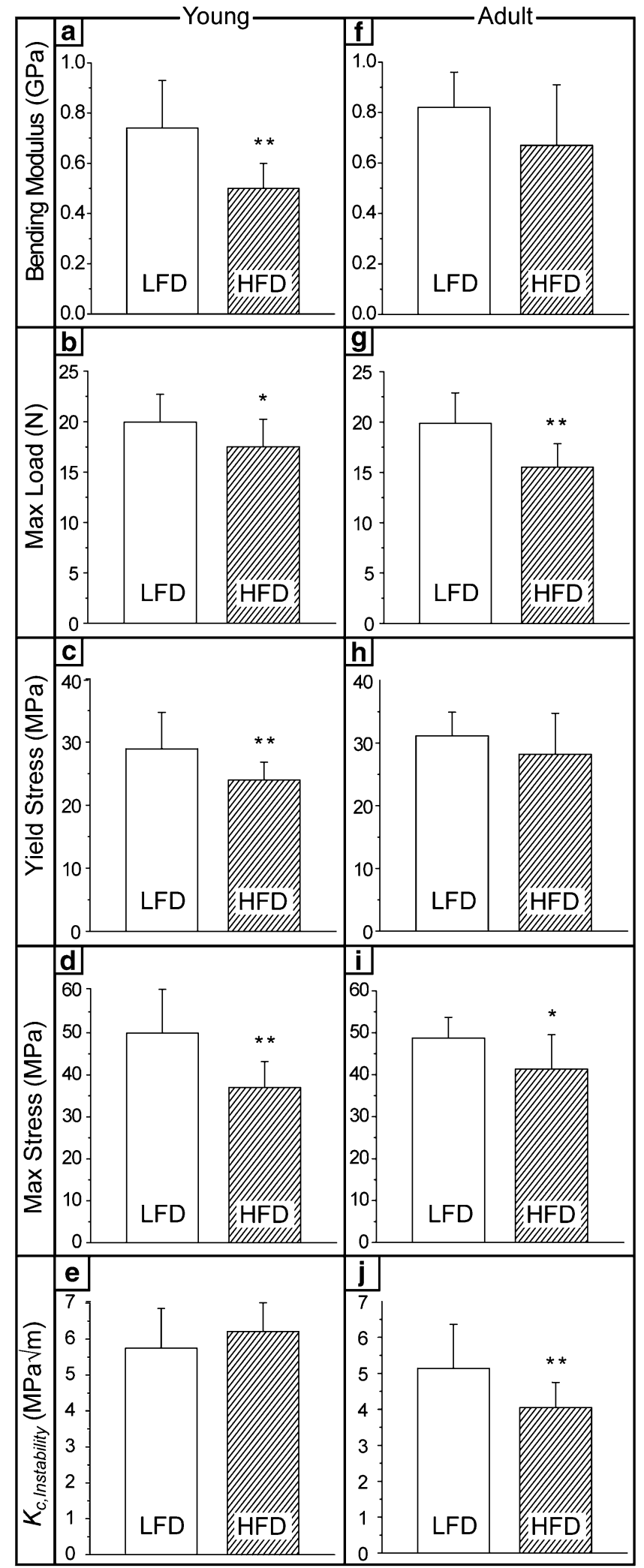


Fig. 5 SEM images of the fracture region showing cortical bone tissue structure changes at the posterior region. a yLFD group; b yHFD; c aLFD; d aHFD. The scale bar indicates $20 \mu \mathrm{m}$. The posterior cortex in HFD bone in (b) and (d) shows reduced alignment of osteocyte lacunae and reduction in lamellar alignment at the tissue level. These images are representative of three samples each of aHFD, yHFD, aLFD, and yLFD. Medial, lateral, and anterior portions of the bone sections appeared similar for HFD and LFD in both age groups

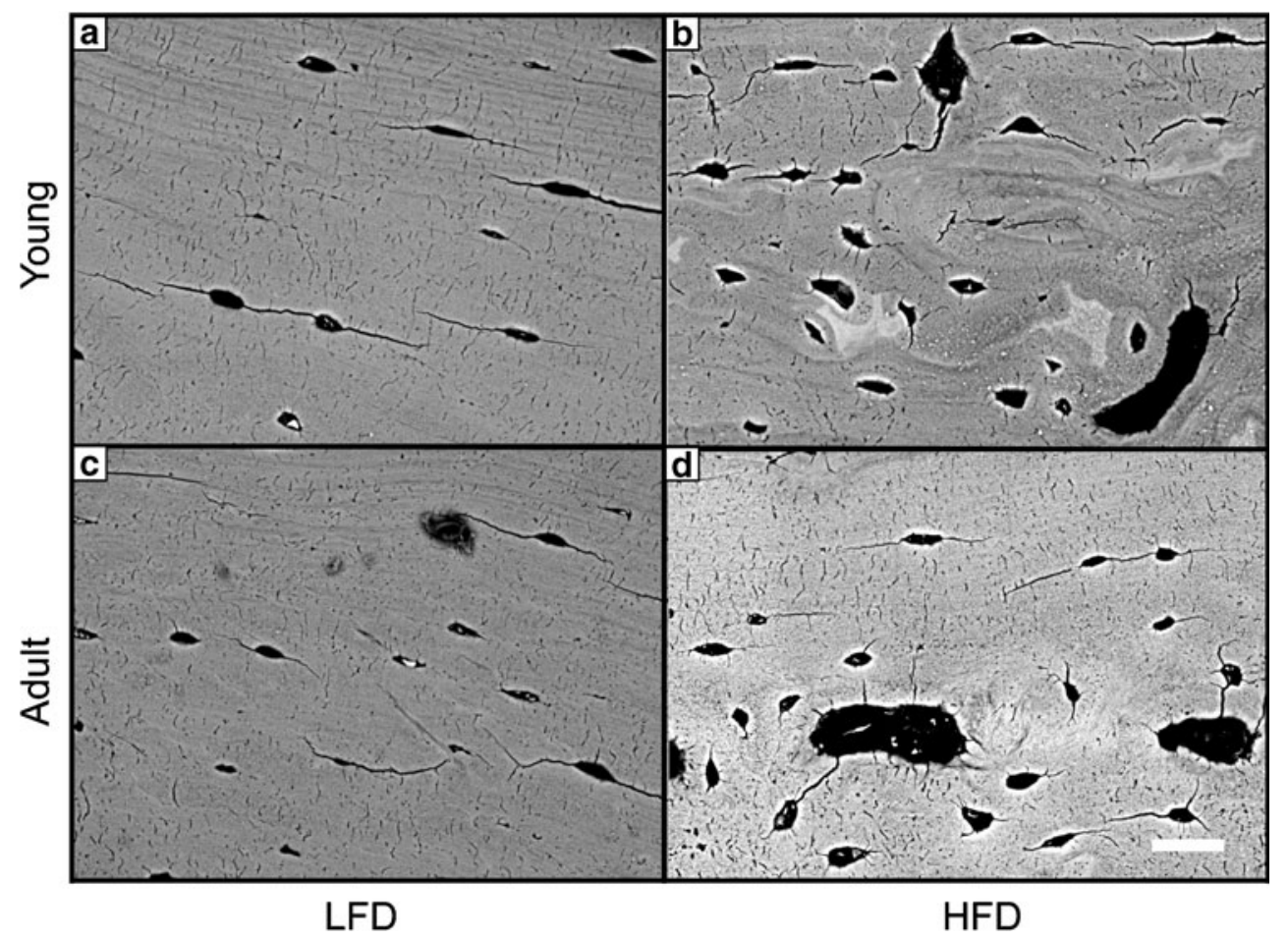

Correlation analysis: bone size measures, rather than body composition or bone mineral measures, most predictive of mechanical properties

In order to evaluate relationships between bone size, mechanical properties, and body composition, correlation analyses were performed between scores for each measure; correlation coefficients and corresponding $p$ values are summarized in Table $1(\mathrm{Q}-\mathrm{Q}$ analysis revealed that the data were normally distributed). In the aLFD, yLFD, aHFD, and yHFD groups, bone size measures have the highest negative correlation coefficients with size-independent mechanical measures, although significance was more difficult to achieve in the HFD groups. The next highest predictor of mechanical properties appears to be LBM, which is not surprising as bone size is highly positively correlated with LBM. FBM had a weak but negative correlation with bone size measures, and therefore appears to have little effect on mechanical properties. BMC affected mechanical properties more than aBMD, but aBMD is confounded with bone size. A size-independent measure of BMD such as volumetric BMD (vBMD) may show a stronger correlation between mineral distribution and mechanical properties. Interestingly, size-independent measures of bone quality (strength, fracture toughness) are most affected by the size of the bone, which implies a reduced quality with increasing quantity even in the nonobese groups.

\section{Discussion}

In this study, we have evaluated the effects of diet-induced obesity on cortical bone and found a large reduction in the mechanical properties of the cortical bone with diabetic obesity in both young and adult mice. Although larger bone size is expected, especially with higher lean body mass [26, 36-39], the mechanical performance of the bone is nevertheless degraded by the effects of obesity with higher leptin and IGF-I levels and significantly higher fat body mass. As higher IGF-I levels are associated with larger bone size, especially at the periosteum, these data are in agreement with our observed trends in bone size in the young group. The slight reduction in IGF-I for adults is also in agreement with the slight reduction in bone size that was observed in aHFD. Such reduced mechanical properties are also consistent with the high blood glucose levels, which may be a partial contributor to the fracture incidence observations in diabetic people [4, 13]. Finally, the greater AGEs with obesity may offer insight into the observed reduced mechanical properties. Assuming that the levels of AGEs are normal in the LFD groups, then the elevated levels in the HFD groups could help explain reduced fracture toughness [23-25], especially in the adult group, as the resultant increase in collagen cross-linking can suppress plasticity in bone by such mechanisms as fibrillar sliding.

We specifically investigated changes in both tissue quantity, as measured by bone size and mineral content, 
Table 1 Correlation coefficients between standardized properties in bone from (a)-(d) young and (e)-(h) adult groups

\begin{tabular}{|c|c|c|c|c|c|c|}
\hline \multirow[t]{3}{*}{ Predictors } & \multicolumn{3}{|c|}{ a. Young LFD $(n=15)$} & \multicolumn{3}{|c|}{ b. Young HFD $(n=15)$} \\
\hline & \multicolumn{2}{|c|}{ Size-independent measures } & \multirow{2}{*}{$\begin{array}{l}\text { Size-dependent measures } \\
P_{u}\end{array}$} & \multicolumn{2}{|c|}{ Size-independent measures } & \multirow{2}{*}{$\begin{array}{l}\text { Size-dependent measures } \\
P_{u}\end{array}$} \\
\hline & $\left(\sigma_{y}, \sigma_{u}, E\right)$ & $K_{c}$ & & $\left(\sigma_{y}, \sigma_{u}, E\right)$ & $K_{c}$ & \\
\hline aBMD & -0.3357 & 0.2225 & 0.3055 & 0.0317 & $0.5767^{*}$ & 0.5089 \\
\hline $\mathrm{BMC}$ & -0.2654 & 0.3362 & 0.4731 & 0.1793 & 0.4383 & 0.2907 \\
\hline (D, t, M.A.) & $-0.7497 * *$ & 0.4931 & 0.1384 & -0.4951 & 0.0037 & 0.214 \\
\hline LBM & -0.4108 & 0.319 & 0.3969 & -0.2584 & 0.0167 & 0.1194 \\
\hline \multirow[t]{2}{*}{ FBM } & 0.1384 & -0.2299 & -0.1014 & 0.1582 & -0.4439 & -0.2404 \\
\hline & \multicolumn{3}{|c|}{ c. Bone size in LFD-(D, t, M.A.) } & \multicolumn{3}{|c|}{ d. Bone size in HFD-(D, $t$, M.A. $)$} \\
\hline LBM & \multicolumn{3}{|c|}{$0.8133^{* * *}$} & \multicolumn{3}{|l|}{0.4982} \\
\hline FBM & \multicolumn{3}{|l|}{-0.1433} & \multicolumn{3}{|l|}{-0.4298} \\
\hline \multirow[t]{3}{*}{ Predictors } & \multicolumn{3}{|c|}{ e. Adult LFD $\left(n=13^{\mathrm{a}}\right)$} & \multicolumn{3}{|c|}{ f. Adult HFD $(n=14)$} \\
\hline & \multicolumn{2}{|c|}{ Size-independent measures } & Size-dependent measures & \multicolumn{2}{|c|}{ Size-independent measures } & Size-dependent measures \\
\hline & $\left(\sigma_{y}, \sigma_{u}, E\right)$ & $K_{c}$ & $P_{u}$ & $\left(\sigma_{y}, \sigma_{u}, E\right)$ & $K_{c}$ & $P_{u}$ \\
\hline aBMD & 0.0808 & 0.2741 & 0.0574 & -0.4976 & 0.2376 & -0.2333 \\
\hline $\mathrm{BMC}$ & -0.1709 & 0.1131 & 0.3577 & -0.4312 & -0.0746 & -0.0991 \\
\hline (D, t, M.A.) & $-0.5559^{*}$ & 0.3858 & $0.7536^{*}$ & -0.5046 & -0.3889 & 0.4426 \\
\hline LBM & 0.1485 & 0.3775 & 0.5138 & -0.2061 & -0.1537 & $0.6519^{*}$ \\
\hline \multirow[t]{2}{*}{ FBM } & -0.1075 & 0.0715 & -0.4535 & -0.1394 & -0.3774 & -0.0796 \\
\hline & \multicolumn{3}{|c|}{ g. Bone size in LFD-(D, t, M.A.) } & \multicolumn{3}{|c|}{ h. Bone size in HFD-(D, t, M.A.) } \\
\hline LBM & \multicolumn{3}{|l|}{0.4587} & \multicolumn{3}{|l|}{$0.6377^{*}$} \\
\hline FBM & \multicolumn{3}{|l|}{-0.1284} & \multicolumn{3}{|l|}{-0.0023} \\
\hline
\end{tabular}

Coefficients from correlation analysis applied between standardized mechanical properties and standardized bone and physiological properties of (a), (c) young LFD group; (b), (d) young HFD group; (e), (g) adult LFD group; (f), (h) adult HFD group. In cases where measurements were related and highly positively correlated, a composite score was used in the analysis. Bone size is the largest predictor of mechanical properties, more so than bone mineral measures or body composition. Interestingly, size-independent measures of bone quality are most affected by the size of the bone, which implies a reduced quality with increasing quantity. Correlation coefficients between body mass measures and bone size measures show that LBM is positively correlated with bone size in both groups (c), (d), (g), (h) and that FBM is very weakly negatively correlated with bone size. Correlation coefficients are conducted separately for young and adult groups

$\nu B M D$ volumetric bone mineral density, $M . A$. second moment of area, $A$ Ct. cross-sectional area, $R_{o}$ outer Ct. Rd, $L B M$ lean body mass, $F B M$ fat body mass, $\sigma_{y}$ yield strength, $\sigma_{u}$ maximum strength, $E$ bending modulus, $K_{c}$ fracture toughness, $P_{y}$ yield load, $P_{u}$ maximum load, $(D, t, M . A$.) composite bone size score, $\left(\sigma_{y}, \sigma_{u}, E\right)$ composite strength and modulus score

${ }^{*} p<0.05,{ }^{* *} p<0.01,{ }^{* * *} p<0.001$

${ }^{\text {a }}$ One mouse died in week 4 of the study from fighting

and bone tissue quality, which was quantified with histomorphometric analyses and qualified by imaging of structural organization. Geometric effects were small (young mice had increased diameter, adult mice had reduced cortical thickness, and other measures were unchanged). The finding of reduced bone quality is supported by SEM studies which reveal reduced tissue organization at the micro- and nano-scale and reduced alignment of lamellae, especially in the posterior quadrant. Both relatively unchanged bone size and decreasing quality of tissue suggest that the bone would be less able to perform its loadbearing function. The reduced ability of bone to bear loads is supported by large reductions in both the size-dependent and size-independent mechanical measures. Overall, we see a reduction of bone tissue quality with minor changes in tissue quantity (bone size measures) in both adult and young mice. Correlation analysis supports this finding as size-independent measures of bone quality (strength, fracture toughness) are most affected by the size of the bone, which implies a reduced quality with greater quantity even in the non-obese groups. There are, however, differences between the two age groups in their response to obesity, which this work addressed by considering the effects of diabetic obesity at two stages of an age spectrum.

Additionally, there are changes in bone response to diabetic obesity with age. Obese adults had smaller femoral thickness than control adults, while the obese young had larger femoral diameter compared to young controls. This shift is supported by greater serum IGF-I concentrations in young mice. Although not significant, it is possible that age 
decreases the ability of bones to increase in size in response to increasing obesity. This inability of bone size to respond to increased weight coupled with the observed degraded mechanical properties suggests that adults are just as at risk for bone fracture, if not more so, than the young group when diabetes is present. These findings in a mouse model agree with human fracture rates, which increase in diabetic obesity for both young and adults $[4,13]$.

This study is limited in that markedly greater blood glucose levels were observed, and this potential diabetic state likely interferes with the body's tendency to increase bone size in response to increasing leptin, IGF-I, and body weight as would otherwise be expected. Our results support those of Garris et al. who found reduced hind limb bone maturation in $\mathrm{db} / \mathrm{db}$ (diabetic) and ob/ob (obese) mice relative to controls [40]. Our prior study [19], which used a different low-fat diet but the same high-fat diet, found a smaller effect on blood glucose levels over a longer period of time (19 weeks) and also a much larger effect on bone size (markedly greater cortical bone parameters). It is therefore highly likely that the differences in the two studies (i.e., reduced effect in bone size, whereby cortical size parameters seem to be relatively unchanged by obesity in this work) results from the additional burden of diabetes. Studying mouse models that are less susceptible to hyperglycemia may show larger effects in the bone size such as those observed in nondiabetic humans. Additional study is warranted to investigate how the findings in this study are reflected in humans.

Overall, we observed a marked reduction in mechanical performance of cortical bone in both adults as well as adolescents with obesity. Modest bone size changes were observed, although the trend appears to change from greater bone size in young obese mice to smaller bone size in adult obese mice as compared to their respective lean controls. Both the bone size and surface-based bone turnover investigations are in agreement with the reversing serum IGF-I concentration, smaller in young and trending larger in adults. These observations are in agreement with human fracture incidence data where increasing fracture rates accompany diabetic obesity. Factors such as hormone levels and blood glucose levels dramatically influence the effects of obesity on bone, and may even cancel out the compensatory mechanisms such as the tendency of bone to increase its size in response to increasing body size.

Acknowledgments This study was supported by the Laboratory Directed Research and Development Program of Lawrence Berkeley National Laboratory (LBNL), funded by the U.S. Department of Energy under contract no. DE-AC02-05CH11231 (for SSIM, JWA III, ROR). Animal study work was supported by the National Institutes of Health (NIH) under grant nos. RO1-DE019284 (for TA) and RO160540, 68152 (for JMW, CV), as well as the American Heart Association, grant nos. CDA $740041 \mathrm{~N}$ (for JMW, CV) and $0825215 \mathrm{~F}$ (for JMW). Bone histomorphometry was supported by
NIH grants RO1-AR43052, AR048841 (for MS, WY, NEL). AGE accumulation analysis was supported by NIH grant no. F32-059497-01 (for ST). We acknowledge the laboratories of R. Ramesh at UC Berkeley and S. Robinson at Beckman Institute (UI Urbana-Champaign, IL) where the SEM work was performed.

\section{Conflicts of interest None.}

Open Access This article is distributed under the terms of the Creative Commons Attribution Noncommercial License which permits any noncommercial use, distribution, and reproduction in any medium, provided the original author(s) and source are credited.

\section{References}

1. Flegal KM, Carroll MD, Ogden CL, Johnson CL (2002) Prevalence and trends in obesity among US adults, 1999-2000. JAMA 288:1723-1727

2. Kopelman PG (2000) Obesity as a medical problem. Nature 404:635-643

3. Taylor ED III, Theim KR, Mirch MC, Ghorbani S, TanofskyKraff M, Adler-Wailes DC, Brady S, Reynolds JC, Calis KA, Yanovski JA (2006) Orthopedic complications of overweight in children and adolescents. Pediatrics 117:2167-2174

4. Lipscombe LL, Booth GL, Jamal SA, Hawker GA (2007) The risk of hip fractures in older individuals with diabetes. Diabetes Care 30:834-841

5. Edelstein SL, Barrett-Connor E (1993) Relation between body size and bone mineral density in elderly men and women. Am J Epidemiol 138:160-169

6. Glauber HS, Vollmer WM, Nevitt MC, Ensrud KE, Orwoll ES (1995) Body weight versus body fat distribution, adiposity, and frame size as predictors of bone density. J Clin Endocrinol Metab 80:1118-1123

7. Reid IR (2008) Relationships between fat and bone. Osteoporos Int 19:595-606

8. Albala C, Yanez M, Devoto E, Sostin C, Zeballos L, Santos JL (1996) Obesity as a protective factor for postmenopausal osteoporosis. Int J Obesity 20:1027-1032

9. De Laet C, Kanis JA, Oden A, Johanson H, Johnell O, Delmas P, Eisman JA, Kroger H, Fujiwara S, Garnero P, McCloskey EV, Mellstrom D, Melton LJ 3rd, Meunier PJ, Pols HAP, Reeve J, Silman A, Tenenhouse A (2005) Body mass index as a predictor of fracture risk: a meta-analysis. Osteoporosis Int 16:1330-1338

10. Hills AP, Parker AW (1992) Locomotor characteristics of obese children. Child Care Health Dev 18:29-34

11. Colne P, Frelut ML, Peres G, Thoumie P (2008) Postural control in obese adolescents assessed by limits of stability and gait initiation. Gait Posture 28:164-169

12. Wang L, Li JX, Xu DQ, Hong YL (2008) Proprioception of ankle and knee joints in obese boys and nonobese boys. Med Sci Monit 14:CR129-CR135

13. Saha MT, Sievanen H, Salo MK, Tulokas S, Saha HH (2009) Bone mass and structure in adolescents with type 1 diabetes compared to healthy peers. Osteoporos Int 20:1401-1406

14. Brahmabhatt V, Rho J, Bernardis L, Gillespie R, Ziv I (1998) The effects of dietary-induced obesity on the biomechanical properties of femora in male rats. Int J Obesity 22:813-818

15. Li KC, Zernicke RF, Barnard RJ, Li AFY (1990) Effects of a high-fat sucrose diet on cortical bone morphology and biomechanics. Calcif Tissue Int 47:308-313 
16. Zernicke RF, Salem GJ, Barnard RJ, Schramm E (1995) Longterm, high-fat-sucrose diet alters rat femoral neck and vertebral morphology, bone mineral content, and mechanical properties. Bone 16:25-31

17. Kawashima Y, Fritton JC, Yakar S, Epstein S, Schaffler MB, Jepsen KJ, LeRoith D (2009) Type 2 diabetic mice demonstrate slender long bones with increased fragility. Bone 44:648-655

18. Turner CH, Burr DB (1993) Basic biomechanical measurements of bone: a tutorial. Bone 14:595-608

19. Ionova-Martin SS, Do SH, Barth HD, Szadkowska M, Porter AE, Ager JW III, Ager JW, Alliston T, Vaisse C, Ritchie RO (2010) Reduced size-independent mechanical properties of cortical bone in high-fat diet-induced obesity. Bone 46:217-225

20. Karsenty G (2006) Convergence between bone and energy homeostases: leptin regulation of bone mass. Cell Metab 4: 341-348

21. He J, Rosen CJ, Adams DJ, Kream BE (2006) Postnatal growth and bone mass in mice with IGF-I haploinsufficiency. Bone 38: 826-835

22. Bluher M, Kahn BB, Kahn R (2003) Extended longevity in mice lacking the insulin receptor in adipose tissue. Science 299:572-574

23. Vashishth D, Wu P, Gibson GJ (2004) Age-related loss in bone: toughness is explained by non-enzymatic glycation of collagen. Transactions of the 50th Annual Meeting of the Orthopaedic Research Society. \#497, San Francisco, CA.

24. Vashishth D, Gibson GJ, Khoury JI, Schaffler MB, Kimura J, Fyhrie DP (2001) Influence of nonenzymatic glycation on biomechanical properties of cortical bone. Bone 28:195-201

25. Bailey AJ, Paul RG, Knott L (1998) Mechanisms of maturation and ageing of collagen. Mech Ageing Dev 106:1-56

26. Petit MA, Beck TJ, Shults J, Zemel BS, Foster BJ, Leonard MB (2005) Proximal femur bone geometry is appropriately adapted to lean mass in overweight children and adolescents. Bone 36:568-576

27. Pollock NK, Laing EM, Baile CA, Hamrick MW, Hall DB, Lewis RD (2007) Is adiposity advantageous for bone strength? A peripheral quantitative computed tomography study in late adolescent females. Am J Clin Nutr 86:1530-1538

28. Leonard MB, Shults J, Wilson BA, Tershakovec AM, Zemel BS (2004) Obesity during childhood and adolescence augments bone mass and bone dimensions. Am J Clin Nutr 80:514-523
29. Lorentzon M, Landin K, Mellstrom D, Ohlsson C (2006) Leptin is a negative independent predictor of areal BMD and cortical bone size in young adult Swedish men. J Bone Miner Res 21: $1871-1878$

30. Hennenberg M, Ulijaszek SJ (2010) Body frame dimensions are related to obesity and fatness: lean trunk size, skinfolds, and body mass index. Am J Hum Biol 22:83-91

31. Parfitt AM, Drezner MK, Glorieux FH, Kanis JA, Malluche H, Meunier PJ, Ott SM, Recker RR (1987) Bone histomorphometry: standardization of nomenclature, symbols, and units. Report of the ASBMR Histomorphometry Nomenclature Committee. J Bone Miner Res 2:595-610

32. Woessner JF (1961) The determination of hydroxyproline in tissue and protein samples containing small proportions of this imino acid. Arch Biochem Biophys 93:440-447

33. Ritchie RO, Koester KJ, Ionova S, Yao W, Lane NE, Ager JW (2008) Measurement of the toughness of bone: a tutorial with special reference to small animal studies. Bone 43:798-812

34. Akhter MP, Cullen DM, Gong G, Recker RR (2001) Bone biomechanical properties in prostaglandin EP1 and EP2 knockout mice. Bone 29:121-125

35. Ferguson VL, Ayers RA, Bateman TA, Simske SJ (2003) Bone development and age-related bone loss in male C57BL/6J mice. Bone 33:387-398

36. Burr DB (1997) Muscle strength, bone mass, and age-related bone loss. J Bone Miner Res 12:1547-1551

37. Janicka A, Wren TAL, Sanchez MM, Dorey F, Kim PS, Mittelman SD, Gilsanz V (2007) Fat mass is not beneficial to bone in adolescents and young adults. J Clin Endocrinol Metab 92:143-147

38. Petit MA, Beck TJ, Lin HM, Bentley C, Legro R, Lloyd T (2004) Femoral bone structural geometry adapts to mechanical loading and is influenced by sex steroids: the Penn State Young Women's Health Study. Bone 35:750-759

39. Wu S, Lei SF, Chen XD, Tan LJ, Jian WX, Xu H, Deng FY, Sun X, Xiao SM, Jiang C, Guo YF, Zhu XZ, Deng HW (2007) The contributions of lean tissue mass and fat mass to bone geometric adaptation at the femoral neck in Chinese overweight adults. Ann Hum Biol 34:344-353

40. Garris DR, Burkemper KM, Garris BL (2007) Influences of diabetes $(\mathrm{db} / \mathrm{db})$, obese (ob/ob) and dystrophic (dy/dy) genotype mutations on hind limb maturation: a morphometric, radiological and cytochemical indices analysis. Diabetes Obes Metab 9: 311-322 